\title{
Initial Experience in Moving Key Academic Department Functions to Social Networking Sites
}

\author{
David Dennis \\ Loyola University Chicago, ddennis@luc.edu \\ George K. Thiruvathukal \\ Loyola University Chicago, gkt@cs.luc.edu \\ Konstantin Läufer \\ Loyola University Chicago, klaeufer@gmail.com
}

Follow this and additional works at: https://ecommons.luc.edu/cs_facpubs

Part of the Computer Sciences Commons

\section{Recommended Citation}

David Dennis, Konstantin Läufer, and George K. Thiruvathukal, "Initial experience in moving key academic department functions to social networking sites", In Proc. 6th International Conference on Software and Data Technologies (ICSOFT) (July 2011)

This Conference Proceeding is brought to you for free and open access by the Faculty Publications and Other Works by Department at Loyola eCommons. It has been accepted for inclusion in Computer Science: Faculty Publications and Other Works by an authorized administrator of Loyola eCommons. For more information, please contactecommons@luc.edu. (c) $($ ) $(9)$

This work is licensed under a Creative Commons Attribution-Noncommercial-No Derivative Works 3.0 License. Copyright @ 2011 David Dennis, Konstantin Läufer, and George K. Thiruvathukal 


\title{
INITIAL EXPERIENCE IN MOVING KEY ACADEMIC DEPARTMENT FUNCTIONS TO SOCIAL NETWORKING SITES
}

\author{
David B. Dennis ${ }^{1}$, Konstantin Läufer ${ }^{2}$ and George K. Thiruvathukal ${ }^{2}$ \\ ${ }^{1}$ Department of History, Loyola University Chicago, Chicago, IL 60626, USA \\ ${ }^{2}$ Department of Computer Science, Loyola University Chicago, Chicago, IL 60611, USA \\ dennis@luc.edu, \{laufer,gkt\}@cs.luc.edu
}

Keywords: academic computing, cloud computing, collaboration, course management, groupware, software-as-a-service

\begin{abstract}
We discuss our initial experience with the transition from conventional technology to social networking sites and other cloud-backed sites for three core business functions of two academic departments, of computer science and history, at a mid-size private university: course management, research and administrative collaboration, and community engagement. We first discuss the social/cultural context that informs our technology choices, as well as the evolution of the technology choices themselves. Then, we identify the targeted department functions and their actors. Next, we describe the past and present technical architectures used to support these functions. We conclude with a discussion of our preliminary experience with this transition and to what extent our experience can be generalized into a blueprint that can be adopted by other organizations.
\end{abstract}

\section{INTRODUCTION}

Academic departments at colleges and universities perform various functions that involve collaboration among the members of its community and efforts to expand the community by attracting new students and, if positions are available, new faculty and staff. To perform these functions as effectively as possible, departments choose processes and technologies that support these goals and are appropriate for the targeted groups of users. Making these choices can benefit from an understanding of both the technologies themselves and the digital culture of its users.

As new technologies emerge and digital culture evolves, academic departments need to decide which technologies to adopt and when to implement them in order to continue functioning effectively. We report on our experience with the recent transition of three central department functions from conventional approaches to new ones based on hosted social networking sites (Weaver and Morrison, 2008) and other cloud-backed sites (Lenk et al., 2009):

- course management by faculty for their classes

- research collaboration among faculty, students, and external collaborators

- engagement with the community served by the department, including the dissemination of departmental announcements, enabling students and alumni to network, and other forms of interaction
In the remainder of this paper, we first discuss the social/cultural context that informs our technology choices (Brandtzæg and Heim, 2009), as well as the evolution of the choices themselves (Weaver and Morrison, 2008). Then, we identify the targeted department functions and their actors. Next, we describe the past and present technical architectures used to support these functions. Finally, we discuss our preliminary experience with this transition and to what extent our experience can be generalized.

\section{SOCIAL CONTEXT}

It is useful to consider technology within the social/cultural context in which it is used. In particular, making technology choices can benefit from an understanding of the digital culture of the actors involved. Digital culture has evolved in four major stages among each of the post-WWII generations.

Baby Boomers (1946-1964) This generation of post-WWII births was raised with various types of print and broadcast media, as well as postal mail and the telephone for communication. The internet emerged as a mainstream technology after this generation was well into adulthood (Nayak et al., 2010).

Generation X (1965-1981) This post-baby-boom generation was the first to have widespread 
access to television during its formative years and experienced. Mobile phones and the internet emerged as mainstream technology when members of this generation were teenagers or in their twenties (Tapscott, 1999).

Generation Y (1982-1995) This generation was born into the later stages of the personal computing era and the early internet, before broadband became widely available. Its members embraced mobile phones and social networking as teenagers or young adults (Tapscott, 1999).

Generation Z (1996-present) This "Internet Generation of Digital Natives" is being born into a world of mobile smart phones connected through social networking (Tapscott, 2009).

The boundaries among generations are blurred, and many individuals fall into more than one generation in terms of their technology-related behavior and habits.

As we learned in our departments, engaging the different generations can be challenging (Pfeil et al., 2009; Waycott et al., 2009). Baby boomers and even gen-Xers tend to be suspicious of new technologies as fad, whereas gen-Yers are more open, and gen-Zers appear to be fully digital in orientation. More importantly, gen-Yers and gen-Zers are increasingly willing to live more "public" private lives, much more comfortable with sharing their personal data with others.

Given that generation Y represents all current college students, and the Digital Natives of generation $\mathrm{Z}$ are now entering college and will constitute the vast majority of users over the next ten years, this cultural shift will have profound implications for the future of academic management processes.

\section{EVOLVING INTERACTION}

In this section, we describe the transition undergone by the online interaction technology used to support the academic functions outlined above.

\subsection{The Distant Pre-Web Past}

The distant past, by internet standards, refers to the pre-web era. Even though the web had been in existence since 1989 in the form of hyperlinked text documents, this era effectively continued until the wider commercial adoption of the web in the mid-1990s. During this period, various disjointed mechanisms were used to provide content in a read-only fashion: e.g., FTP, Gopher, WAIS, etc.

This period also saw the development of various means of interaction, though these were likewise disassociated. Asynchronous communication with indi- viduals occurred via email. Public and internal discussion was supported by USENET groups. Document submission was provided by FTP upload. Accordingly, online interaction among the various actors described above was limited mostly to email and USENET, and even that was possible only among the few people who had access to these mechanisms.

\subsection{The Recent Web and Email Past}

In the mid-1990s, commercial adoption of the web increased tremendously, and content dissemination was consolidated on the web in the form of read-only sites.

Interactive offerings included interactive discussion fora, some first-generation consumer-oriented websites for photo sharing, driving directions, etc., and some developer-oriented sites for collaborative development. Fueled by the dramatic growth in internet access among the general population, email skyrocketed as the main means of nonpublic interaction.

\subsection{The Social Networking Present}

The early 2000s saw the emergence of a phenomenon often called web 2.0 (O'Reilly, 2007; Jahnke and Koch, 2009). The major change brought about by web 2.0 has been to engage users in the creation and classification of content. Examples are community-based content creation efforts such as Wikipedia and the widespread use of tags instead of preset categories.

Furthermore, while first-generation interactive websites have been designed as silos that required screen-scraping for programmatic interaction, most second-generation functionality is service-oriented and exposes a documented API for programmatic interaction (Weiss and Gangadharan, 2010). This change is technologically significant because it allows individual applications to focus on performing fewer functions better and supporting cross-integration.

The middle of this past decade gave birth to two related trends: social networking and cloud computing. The former typically are software-as-a-service (SaaS) offerings in cloud terminology. Based on the SaaS paradigm, a wide range of well-known cloudbacked offerings have emerged, such as:

- general social networking sites such as Facebook and Orkut

- specialized professional networking sites such as LinkedIn and XING

- hosted conventional and lightweight blogs such as Blogger and Tumblr, respectively

- microblogging sites such as Twitter

- social bookmarking sites such as Delicious and StumbleUpon 
- web content management sites such as Google Sites

- online document management and groupware sites such as Google Docs and Zoho

- social coding sites such as Bitbucket and GitHub

\section{TARGETED FUNCTIONS}

Our study of how recent and ongoing transition to technologies based on hosted social-networking has impacted management of academic affairs focuses on the following three fundamental department functions. In this section, we briefly define these functions and the associated workflows, scenarios, and actors.

\subsection{Course Management}

Course management is the process used by faculty to manage the various aspects of teaching a course to a class of students (Gauthier and Impey, 2004). This function is complex but consists of several wellunderstood scenarios.

- dissemination of course materials

- group discussions

- submission and evaluation of written homework

- submission and evaluation of code examples

- student presentations and their evaluation

- test taking and evaluation

- sharing grades with students

- conducting polls on students

\subsection{Research Collaboration}

Research collaboration among computer science faculty, students, and external collaborators can be described as a workflow that involves the following activities (Kraut et al., 1987; Ding et al., 2010).

1. knowledge gathering

2. project planning

3. collaborative writing and software development

4. bibliography management

5. formal publication

6. other types of dissemination

\subsection{Community Engagement}

The community served by a department includes its current students, faculty, and staff, prospective students, alumni (former students), employers, candidates for faculty or staff positions, as well as more loosely connected groups such as the parents of current students. The complex, amorphous function of engaging this community comprises several scenarios, including the following (Waters et al., 2009).

- maintaining the department's official public web content

- maintaining the department's internal content (intranet)

- facilitating the department's internal processes, such as faculty meetings, committee decisions, etc.

- enabling faculty to maintain web content specific to their teaching and research activities

- disseminating of various types of departmental announcements to current and former students

- enabling current students to network with each other and departmental faculty and staff

- enabling alumni (former students) to network with each other and departmental faculty and staff

- disseminating entry-level job postings to current students

- disseminating advanced job postings to alumni

\subsection{Actors}

The following actors are involved in these workflows and scenarios:

Faculty in their roles as teachers and researchers belong mostly to the baby boomer and $\mathrm{X}$ generations. As computer scientists and scholars exposed to university information technologies, they tend to be more technology-savvy than other members of these generations but still display some of their characteristics, such as privacy concerns, reluctance to participate in social networking, etc. Younger faculty are being hired only very slowly in these departments.

Staff in their job-specific roles such as secretary, system administrator, etc. are predominantly baby boomers and gen-Xers. They tend to understand the technology they use to perform their jobs, but in many cases require training-especially in the case of upgrades or new systems.

Current students, undergraduate and graduate alike, almost overwhelmingly belong to generation Y. Most members of these groups are 
regular social networking users and have some expectations that the organizations they interact with participate in social networking as well.

Prospective students will overwhelmingly belong to generation $\mathrm{Z}$ and beyond. They will have even higher expectations of managing their interactions through social networking. In particular, they will expect their initial engagement with academic institutions or departments to occur through social networking tools (Lindbeck and Fodrey, 2010).

Alumni (former students) span multiple generations and use social networking accordingly. They are particularly interested in networking for social reasons or in search of employment and will engage in technology that supports these goals (Goetchius, 2008).

External users, such as employers, candidates for faculty or staff positions, students' parents, etc., are a highly diverse group in terms of age and level of education. Nevertheless, as these individuals belong to more and more recent generations, their expectations of using social networking for their interactions with academic institutions or departments will continue to increase.

\section{EVOLVING ARCHITECTURE}

In this section, we describe how our technical architecture in support of the above-mentioned targeted department functions have evolved along with the available technologies themselves.

\subsection{Course Management}

In the distant pre-web past, there was very little course management technology available. Instructors provided printed copies of their course materials, collected homework and project submissions on paper and/or removable media (floppy disks), and posted grades on their office doors. Using email to interact with students was then considered an innovation.

In the more recent past, the central Information Technology Services unit at our university acquired a major commercial course management system (Gauthier and Impey, 2004) that was intended to provide all required functionality online. Although acceptance was widely encouraged and continual training has been provided, the system itself has been criticized by numerous faculty for its inflexibility, poor usability, regular and enforced "course deletion" requirements (owing to space limits) and occasional data loss (often associated with the preceding).
At present, the authors have abandoned the central system in favor of a combination of several carefully chosen, free, partially integrated "best-of-breed" cloud-backed technologies:

- Dissemination of course materials occurs via cloud-backed solutions such as Google Sites and hosted blogs.

- Group discussions take place in Google Groups or closed Facebook groups.

- Submission of code examples takes place through social coding sites such as Bitbucket, which provide shared private code repositories that support standard version control software such as Mercurial. In addition, these sites allow students to create a project wiki, set up an issue tracker, etc.

- Written work is submitted through Google Docs.

- Gradebook functionality is provided through Google Docs spreadsheets. Summer work is in progress to have a gradebook with support for various roles, i.e., instructor, student, TA.

- Some online test taking and polls are conducted using Google Forms.

The authors have already been following this approach on several of their sites for computer science and history courses.

\subsection{Research Collaboration}

In the distant past, research collaboration was largely ad-hoc (Kraut et al., 1987; Ding et al., 2010). Code and documents were exchanged by email or removable media, although early version control systems such as SCCS and RCS were used for more systematic collaborative document authoring and code development. Knowledge gathering and interaction took place in meetings, by email, phone, and/or Unix talk.

In the recent past, more modern version control systems such as CVS and Subversion were adopted, often with Secure Shell-based setups that supported collaboration across institutions. Interaction remained largely the same, except that Unix talk was replaced by newer forms of instant messaging.

At present, numerous collaborative research efforts, including the authors' own, have transitioned to a combination of cloud-backed technologies. Specifically, the authors use these (free) systems:

- Knowledge gathering occurs on project-specific Wikis hosted on Google Sites, which supports fine-grained access control.

- Project planning takes place with the help of Basecamp, a simple, effective, and highly usable site.

- Collaborative software development is supported effectively by distributed version control systems 
(DVCS) such as Git or Mercurial. Various social coding sites, such as GitHub and Bitbucket, support these DVCS. We use Bitbucket for nonpublic development because of its free private repositories and GitHub for open-source projects because of its high visibility and social coding features.

- Collaborative writing is the first of two writing phases. In this phase, the authors use Google Docs until they have a coherent draft. Then they switch to an appropriate formal publication mechanism as discussed below.

- Bibliography management can greatly benefit from sharing and centralization. We have identified CiteULike as the most effective option for sharing bibliographic information among collaborators and publicly.

- Before formal publication can occur, suitable venues have to be identified. WikiCFP allows us to manage a list of conferences and workshop to which we plan to submit. Twitter is also useful for receiving announcements from conferences, such as postponed submission deadlines.

- Formal publication itself is the second of two writing phases. In this phase, the authors use LaTeX or LyX to satisfy the envisioned publisher's formatting requirements, and version control systems, as described above, enable collaboration.

- Other types of dissemination include announcing the publication of a paper on a blog such as Tumblr, which can automatically send short versions of posts to Twitter, inclusion in one's CiteULike bibliography, and email to specific individuals. Disqus is a discussion mechanism that integrates with Tumblr, among other sites. Gravatar is a service used by various other sites for mapping of email addresses to avatar pictures. Finally, Google Analytics provides various types of website access data (including blogs) that are useful in estimating the impact of a project or publication.

\subsection{Community Engagement}

In the distant past, community engagement occurred mostly through conventional channels such as mailings, poster advertisements, on-campus meetings, etc. USENET groups also played a role in allowing members of an academic community to interact.

In the recent past, universities attempted to move large parts of their conventional community engagement efforts to web and email, with mixed results. The department of computer science maintains an official website and uses Google Groups for reaching current students by email. There was no systematic way to reach our alumni.
At present, the departments of computer science and history at the authors' institution have established a social networking presence almost overnight by picking and integrating a few specific technologies. Our initial focus has been on engaging students and alumni, which in turn requires that students and alumni be allowed to participate actively and contribute (Waters et al., 2009; Goetchius, 2008).

- To engage our students, we have created a Facebook page with a custom URL that is easy to remember. This page allows our current, former, and prospective students to interact with each other and department faculty informally. This choice was based on the observation that virtually every one of our students already had a Facebook account. We have also set up a Tumblr blog for all public departmental announcements, thereby decentralizing the process of content creation among department faculty. All blog posts automatically feed into a departmental Twitter handle we set up. Discussions are enabled through Disqus. We are currently evaluating how to pipe the blog posts back into the existing Google Groups-based email lists using cloud-backed technology.

- To engage our alumni, we have created a LinkedIn group so as to underscore the professional nature of this effort. We were able to populate this group quickly based on departmental information and one of the authors' personal alumni contacts on LinkedIn and via email. We periodically ask the members of this group to spread the word to other alumni who are not members yet.

\section{CONCLUSION}

We have discussed the way members of the successive postwar social "generations" relate to technology and the evolution of the technology itself. Within this context, we discussed our initial experience with the corresponding technological transition of several core functions of academic departments at a mid-size private university: course management, research collaboration, and community engagement.

Our cloud-backed course management approach has already been used successfully but continues to undergo refinement in technology and process. Our cloud-backed approach to research collaboration has functioned effectively as well and continues to evolve as we evaluate new technologies. We believe that both approaches can already be used as starting points for other departments and research groups.

Our social-networking-based approach to community engagement has been well received and, according to Google Analytics, drawn more traffic to the de- 
partmental website. It has focused on connecting students and alumni so far but needs to be extended to include the various other aspects of this function.

The related work we have surveyed is either descriptive (Lockyer and Patterson, 2008; Hung and Yuen, 2010), focuses on one particular technology (Selwyn, 2009; Roblyer et al., 2010; Ractham and Firpo, 2011), or proposes to build an educational social networking site from scratch (Conole and Culver, 2010). By contrast, our approach focuses on the integration of existing, mature "best-of-breed" sites.

To understand how our observations relate to ongoing efforts by government agencies to broaden the participation in computing, further data is needed on social networking participation across other demographic aspects besides age, such as gender, ethnicity, level of education. In addition, further data on social networking participation across different countries (Mislove et al., 2007) would be useful for generalization across national boundaries.

We conclude by observing that technology continues to evolve very rapidly. Requirements also tend to evolve as the target users change with respect to demographics and use of technology. Therefore information technology decision makers at the various levels of an academic organization need to collaborate closely on requirements and evaluate the available choices very carefully.

\section{REFERENCES}

Brandtzæg, P. and Heim, J. (2009). Why People Use Social Networking Sites. In Ozok, A. A. and Zaphiris, P., editors, Online Communities and Social Computing, volume 5621, chapter 16 . Springer, Berlin.

Conole, G. and Culver, J. (2010). The design of Cloudworks: Applying social networking practice to foster the exchange of learning and teaching ideas and designs. Computers \& Education, 54(3):679-692.

Ding, W. W., Levin, S. G., Stephan, P. E., and Winkler, A. E. (2010). The Impact of Information Technology on Academic Scientists' Productivity and Collaboration Patterns. MANAGEMENT SCIENCE, 56(9).

Gauthier, A. J. and Impey, C. D. (2004). Course Management Systems: Traveling Beyond Powerpoint Slides Online. In Bulletin of the American Astronomical Society, volume 36 of Bulletin of the American Astronomical Society, pages 1499+.

Goetchius, A. (2008). Career building through social networking. Rosen Pub.

Hung, H.-T. and Yuen, S. C. (2010). Educational use of social networking technology in higher education. Teaching in Higher Education, 15(6):703-714.

Jahnke, I. and Koch, M. (2009). Web 2.0 goes academia: does Web 2.0 make a difference? International Journal of Web Based Communities, 5(4):484-500.
Kraut, R. E., Galegher, J., and Egido, C. (1987). Relationships and tasks in scientific research collaboration. Hum.-Comput. Interact., 3(1):31-58.

Lenk, A., Klems, M., Nimis, J., Tai, S., and Sandholm, T. (2009). What's inside the Cloud? An architectural map of the Cloud landscape. In CLOUD '09: Proceedings of the 2009 ICSE Workshop on Software Engineering Challenges of Cloud Computing, volume 0, Washington, DC, USA. IEEE Computer Society.

Lindbeck, R. and Fodrey, B. (2010). Using Technology in Undergraduate Admission: A Student Perspective. Journal of College Admission.

Lockyer, L. and Patterson, J. (2008). Integrating social networking technologies in education: A case study of a formal learning environment. In Proc. Intl. Conf. on Advanced Learning Technologies (ICALT).

Mislove, A., Marcon, M., Gummadi, K. P., Druschel, P., and Bhattacharjee, B. (2007). Measurement and analysis of online social networks. In Proceedings of the 7 th ACM SIGCOMM conference on Internet measurement, IMC '07, New York, NY, USA. ACM.

Nayak, L., Priest, L., and White, A. (2010). An application of the technology acceptance model to the level of Internet usage by older adults. Universal Access in the Information Society, pages 1-8.

O'Reilly, T. (2007). What is Web 2.0: Design Patterns and Business Models for the Next Generation of Software. Social Sci. Research Network Working Paper Series.

Pfeil, U., Arjan, R., and Zaphiris, P. (2009). Age differences in online social networking - A study of user profiles and the social capital divide among teenagers and older users in MySpace. Comput. Hum. Behav., 25(3).

Ractham, P. and Firpo, D. (2011). Using Social Networking Technology to Enhance Learning in Higher Education: A Case Study Using Facebook. In 44th Hawaii Intl. Conf. on System Sciences (HICSS), pages 1-10.

Roblyer, M., McDaniel, M., Webb, M., Herman, J., and Witty, J. V. (2010). Findings on Facebook in higher education: A comparison of college faculty and student uses and perceptions of social networking sites. The Internet and Higher Education, 13(3):134-140.

Selwyn, N. (2009). Faceworking: exploring students' education-related use of Facebook. Learning, Media and Technology, 34(2):157-174.

Tapscott, D. (1999). Growing Up Digital: The Rise of the Net Generation. McGraw-Hill.

Tapscott, D. (2009). Grown Up Digital - How The Net Generation Is Changing Your World. McGraw-Hill.

Waters, R. D., Burnett, E., Lamm, A., and Lucas, J. (2009). Engaging stakeholders through social networking: How nonprofit organizations are using Facebook. Public Relations Review, 35(2):102-106.

Waycott, J., Bennett, S., Kennedy, G., Dalgarno, B., and Gray, K. (2009). Digital divides? Student and staff perceptions of information and communication technologies. Computers \& Education.

Weaver, A. C. and Morrison, B. B. (2008). Social Networking. Computer, 41(2):97-100.

Weiss, M. and Gangadharan, G. R. (2010). Modeling the mashup ecosystem: structure and growth. $R \& D$ Management, 40(1):40-49. 